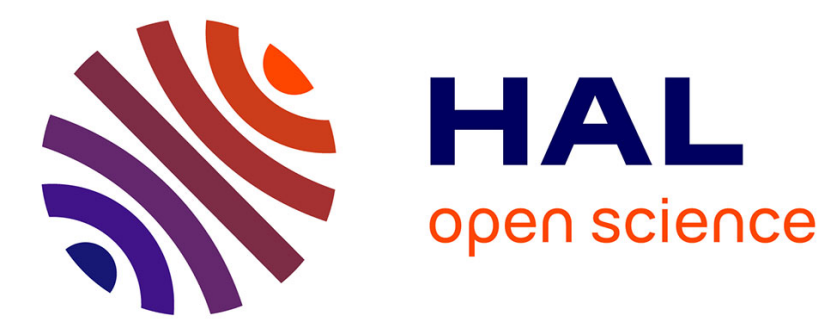

\title{
Collaboration in Virtual Enterprises through the Smart Vortex Suite
}

\author{
M. Biancucci, J. Janeiro, M. Leva, S. Lukosch, M. Mecella
}

\section{To cite this version:}

M. Biancucci, J. Janeiro, M. Leva, S. Lukosch, M. Mecella. Collaboration in Virtual Enterprises through the Smart Vortex Suite. 15th Working Conference on Virtual Enterprises (PROVE), Oct 2014, Amsterdam, Netherlands. pp.426-434, 10.1007/978-3-662-44745-1_42 . hal-01392145

\section{HAL Id: hal-01392145 \\ https://inria.hal.science/hal-01392145}

Submitted on 4 Nov 2016

HAL is a multi-disciplinary open access archive for the deposit and dissemination of scientific research documents, whether they are published or not. The documents may come from teaching and research institutions in France or abroad, or from public or private research centers.
L'archive ouverte pluridisciplinaire HAL, est destinée au dépôt et à la diffusion de documents scientifiques de niveau recherche, publiés ou non, émanant des établissements d'enseignement et de recherche français ou étrangers, des laboratoires publics ou privés.

\section{(c)(1)}

Distributed under a Creative Commons Attribution| 4.0 International License 


\title{
Collaboration in Virtual Enterprises through the Smart Vortex Suite
}

\author{
M. Biancucci ${ }^{1}$, J. Janeiro ${ }^{2}$, M. Leva ${ }^{1}$, S. Lukosch ${ }^{2}$ and M. Mecella ${ }^{1}$ \\ ${ }^{1}$ Sapienza Università di Roma \\ michelebiancucci@gmail.com, \{leva, mecella\}@uniroma1.it \\ ${ }^{2}$ Delft University of Technology \\ \{J.Janeiro, S.G.Lukosch\} @tudelft.nl
}

\begin{abstract}
Collaboration is a key issue in modern virtual enterprises adopting service-oriented business models and functional engineering in the offering of their tangible and intangible assets, as in software oriented IT, CAM/CAD, manufacturing industries. In this paper, we aim at introducing the main components of a Collaboration and Decision Making Suite developed for such scenarios, in particular (i) Elgar, a collaborative workspace supporting the elastic collaboration concept, and a standard interface to realize the integration of groupware tools, and (ii) RegalMiner, capable of capturing, monitoring, processing and visualizing collaboration data streams generated from users of Elgar participating a collaborative process that exploits the elastic collaboration approach in order to evaluate individual and group performances by analyzing the generated activities and the produced text contributions.
\end{abstract}

\section{Introduction}

The last years have witnessed the emergence of a trend in the industry that fosters the offer of intangible assets within vendor-customer relations even if tangible assets still represent a high volume and a very precious part of the delivery. These underlying business models are called "service-oriented models", where the product offer is no longer the equipment itself, but the contractual agreement to specific performances of intangible features like transportation volume, reliability, physical parameters like torque, power, cutting speed or other measurable parameters, which are taken as contractual basis between vendors and customers. This new business model, already used in the software oriented IT and in the CAM/CAD industries, has now found strong interest from the manufacturing companies as well. To ensure that these industries would come into a similar business model, it is required exact knowledge about the behavior of the goods, equipment or the systems, their usage conditions, early detection of upcoming failure modes or maintenance requirements and dependencies with the delivered systems. Additionally, it is required a new way of engineering and design, called "functional engineering", in which a large quantity of data stream is used for validation, behavior prediction, simulation and more in general, will be produced in all phases of the product life cycle. These should be properly analyzed and intelligently managed.

Most important it will be the collaboration support, for improving the product and design life cycle and achieving better decision-making. As systems and products become more complex, and often offered by aggregation of companies (virtual enterprise), experts and engineers of the extended enterprise need to collaborate to manage integration and communication of the subsystems they design and develop. 
Parallel to this, users, clients and stakeholders are becoming increasingly often included in various phases of a product life cycle, including design, user feedback, innovation and improvement cycles. Collaboration in the product's life cycle can be supported by suitable technologies.

The SmartVortex project (cf. www.smartvortex.eu) addresses these topics providing a technological infrastructure consisting of a comprehensive suite of interoperable tools, services, and methods for intelligent management and analysis of massive data streams to achieve better collaboration and decision making in largescale collaborative projects concerning industrial innovation engineering. It consists of a number of software components for capturing, processing and visually query high volume of data streams, together with collaboration and decision making support tools. In particular the infrastructure includes a $(i)$ Data Stream Management System (DSMS), responsible of the access to the data streams and their processing, a (ii) Social Network, that basically realize a cross-organizational middleware which automatically connect organizations seamlessly while still protecting the intellectual property of the partners and minding the different company policies and the (iii) Collaboration and Decision Making Suite, playing a key role in helping the users in the collaborative work regarding data monitoring, data analysis and visualization.

In the following of this paper, we aim at introducing the main components of the Collaboration and Decision Making Suite, in particular (i) Elgar (cf. Section 2), a collaborative workspace supporting the elastic collaboration concept, and a standard interface to realize the integration of groupware tools, and (ii) RegalMiner (cf. Section 3), capable of capturing, monitoring, processing and visualizing collaboration data streams generated from users participating a collaborative process that exploits the elastic collaboration approach in order to evaluate individual and group performances by analyzing the generated activities and the produced text contributions.

\section{Elgar}

In complex design and engineering phases, different tools and techniques are useful at different point in the design. However, groups of engineers often do not have the knowledge to choose or select effective tools and techniques for specific situations. Elgar (ELastic GroupwARe) [8] addresses these problems implementing different coordination mechanism to support groups in collaborative tasks; it provides structured and unstructured coordination.

The major goal of implementing coordination mechanisms in groupware systems is to support users to coordinate task dependencies to accomplish a shared task. On the one side (structured), coordination mechanisms that are highly specified and routinized processes are needed; this allows experts in a certain domain to define processes and structures to support and guide group participants. For example, a software release control system follows a pre-defined process of activities to release new versions of a software product, e.g., bug repair, application testing and final release compilation activities [6]. On the other side (unstructured), coordination mechanisms that are highly unspecified and dynamic processes, are needed in order not use rigid structures or processes to guide group participants in their tasks. Instead, they relies on awareness mechanisms that allow for an informal understanding of the 
activities of others group participants [4]. For example, a spatial visualization of programmers' activities in their development environment and within a project's source code repository system can improve the sense of awareness in a team [3].

Some groupware systems address more than one part of the coordination spectrum and provide mechanisms from both extremes. In such systems, the decision on a specific coordination mechanism is delegated directly to groups of participants, according to the situation they handle [2]. For supply chains, Bernstein proposes a system that implements different coordination mechanisms to enable collaboration between users [2]. The system implements four mechanisms: guidance through scripts, planning based on constraints, monitoring constraints and context provision. Each mechanism supports users to overcome natural unexpected obstacles from the disruption of a computer supply chain, from pre-defined supply chain processes to exploration of context information.
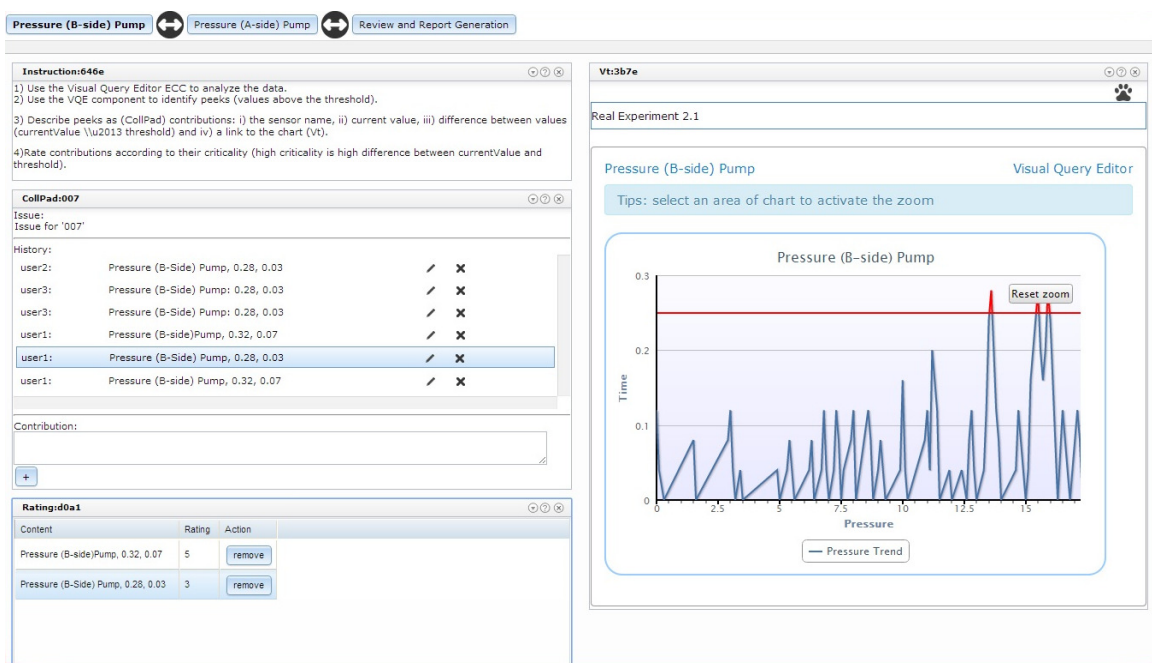

Fig 1. The visualization of Elgar running the prescribed coordination mechanism.

Elgar was implemented as an extensible web-based groupware that provides a modular infrastructure to integrate software components. In Elgar, such components are called Elastic Collaboration Components (ECCs). They represent web-based components, inspired on portlets. Developers need to implement ECCs according to the architecture described in [8] to use them as part of Elgar.

Currently four ECCs have been realized: V2QT, CollPad, Rating and CollPad Categorizer. The Visual Query and Visualization Tool (V2QT) [9] is an ECC that enables users to visually pose continuous queries (CQs) over machine sensor measurements. V2QT handles query requests and responses and displays results in a time-oriented graph. Users gain insights, through the V2QT, over possible anomalous equipment behaviors, e.g. the main hydraulic pressure is above a threshold. 
Collaborative Notepad (CollPad) is a multi-role ECC that allows its users to share their ideas and create text structures, based on lists, collaboratively. The component supports engineers in documenting ideas of possible problems, solutions for given problems, description of consequences for both problems and solutions, and to sketch action plans for a machine's diagnosis process. Such component is important to create collaborative documentation during a diagnosis scenario. The component allows users to define the topic of a certain discussion and provide contributions focusing on the topic. A contribution can either be a category or ideas. Users may combine the CollPad with the V2QT to annotate results of a graph at a particular moment.

Rating is an ECC that allows a user to rate a set of pre-selected contributions from CollPads. Users of Elgar drag contributions from CollPad and drop them in the Rating, creating a dynamic list of contributions collaboratively that will be compared by users through rates. In this component, users can rate the elements of a list according to a scale of five points. The idea is that the highest rated element should be considered for further attention of the group. The rate of contributions is calculated as the average of all rates provided by all participants.

CollPad Categorizer is an ECC that summarizes all discussions in a collaboration session. This component organizes discussions in a list. Each list entry represents a discussion topic, called category, and is automatically associated to a CollPad ECC. Users can contribute to a category by opening the associated CollPad adding, removing or editing content. In addition to the list of categories, users can classify the list of categories with a topic, called issue, which describes the goal of the CollPad Categorizer.

Currently Elgar supports two coordination mechanisms: ad-hoc and prescribed coordination mechanisms.

- Ad-Hoc Coordination Mechanism. The ad-hoc coordination mechanism implements a possible type of unstructured coordination, in which computermediated collaboration within a group naturally emerges and is not guided by prescription. It supports groups that do not need guidance or prescribed coordination during collaboration. The ad-hoc coordination mechanism relies on the use of workspace awareness information to divide and synchronize tasks among group participants. In this mechanism, such information represents all group participants connected in a collaboration session and the ECCs in which participants work. Through such information, participants may create an understanding about activities of others, facilitating their coordination to achieve a common goal, such as in [3]. In addition, this mechanism enables participants to share ECCs with each other to collaborate for content production. Participants can connect to ECCs opened by other participants, provided by workspace awareness information, sharing its content automatically.

- Prescribed Coordination Mechanism. The prescribed coordination mechanism implements a possible type of structured coordination in which professional facilitators plan the sequence of phases and activities. This coordination mechanism guides group participants on the usage of elastic collaboration components. Guidance is described as a collaboration process, which represents a sequence of collaborative phases, each associated with a set of ECCs, and explicit instructions for the phase. In the prescribed coordination mechanism, the collaboration process is described at design time and coordinates the 
collaborative activities, executed by a group. It is based on process-aware systems [5], in which processes are described to coordinate users in accomplishing established goals. The emphasis of the prescribed coordination mechanism is to provide guidance to users in collaborative activities through components, and not to require a facilitator to enable and disable components at specific moments [6].

\section{Regal Miner}

RegalMiner is a collaboration support software tool that is capable of capturing, monitoring, processing and visualizing collaboration data streams generated from users participating a collaborative process, in order to evaluate individual and group performances by analyzing the generated activities and the submitted text contributions, to provide automatic notifications to a third-party facilitator or expert users in case typical meeting problems are detected and to supervise the meeting events to enhance the effectiveness of the current meeting session. The analysis and processing of activities and user contributions is demanded to text mining techniques [1]. RegalMiner adopts a modular approach that mainly consists of five components:

- The Fetcher module is responsible for the communication layer between the groupware platform that outputs the collaboration data streams (i.e., Elgar) and for the storage of all the retrieved information.

- The Reasoner module coordinates the Sentiment Analysis and the Keyword Extraction modules along with the execution of several subtasks:

- sampling the events extracted from the collaboration data stream, according to some predefined intervals that a user, in the role of meeting coordinator, can configure according to the expected time length of the ongoing collaborative process;

- processing of sampled events in order to obtain some basic meeting indicators as Group Participation Rate, Individual Participation Rate, Distribution of Contributions, Number of Contributions, Number of Interactions that provide support for the detection of the levels of attention, interest, discussion and negotiation. The mentioned meeting indicators help diagnose typical meeting problems such as the Dominant Spaces syndrome that occurs when the formation of independent subgroups prevailing on other participants increases the levels of frustration and insulation. Another meeting problem that can be detected by the monitoring of these meeting indicators is the Sleeping Meeting syndrome that is caused by the lack of mental and physical energy thus decreasing the effectiveness of the overall collaborative process;

- cycling the execution of the Sentiment Analysis module that processes the sampled events and returns scores for some high-level meeting indicators as Group Sentiment Analysis Score and Individual Sentiment Analysis Score. These meeting indicators relates to the level of interpersonal agreement or disagreement and individual positioning of participants involved in the collaborative session. Low levels of Sentiment Analysis indicators help to diagnose the Multi-Headed Beast syndrome, that occurs when there is no 
agreement on the agenda and confusion about the problem-solving strategies and participants interrupt or attack each other. Another typical meeting problem that can be detected by monitoring the mentioned scores is the Feuding Factions syndrome caused by internal power struggles that push participants against each other assuming an aggressive body language;

- starting the execution of the Keyword Extraction module whenever the current meeting session has finished or having a break, thus returning the set of Extracted Topics for each participant. The extracted keywords can be associated with the topics and ideas argued during the meeting discussion, especially in brainstorming stages. The results of this process analysis provides insights about the levels of convergence/divergence towards a common solution, idea discussion and idea distinction that can help the early detection of several meeting problems such as Multi-Headed Beast, Dominant Spaces, Sleeping Meeting as already been discussed and Recycling syndrome caused by misunderstandings about the problemsolving processes thus resulting in a lack of consensus among participants that on the consequence start repeating the same arguments and reuse older solutions instead of generating alternative ones;

- The Sentiment Analysis module is in charge of processing the contributions that participants submitted during a meeting session. In particular, it returns the Group Sentiment Analysis Score and Individual Sentiment Analysis Score. It implements a dictionary-based approach built on SentiWordNet [10,7] that has proven to be efficient in terms of computational effort, especially in the case of repeated executions that are the requirement for a online analysis. The Sentiment Analysis module retrieves the collection of contributions for each participant and pre-processes the terms from extracted sentences, then detects terms that imply sense inversion as "no", "not", "don't", etc. and finally looks up into the dictionary to obtain scores for single terms, that are subsequently combined with other scores of terms in the sample of the collaboration data stream. A negative user score near -1 implies that the participant feels uncomfortable, isolated, in disagreement or in contrast with the rest of the participants, which can be one of the symptoms of the Multi-Headed Beast, Feuding Factions or Dominant Spaces syndromes. On the other hand, a positive user score near +1 indicates a user that feels positive, in agreement and an active subject of the group participating the collaborative process. It also reveals a high level of acceptance of group ideas, with a numeric majority of pros in contrast with a numeric minority of cons. Careful attention has to be provided to neutral user scores near 0 that may imply hidden issues that may arise in future phases of the meeting session, since participants that fear changes or are under the influence of a subgroup may not express their real opinions. A facilitator or an expert user is required to thoroughly analyze and understand individual behaviors that happened in the previous stages of the collaborative process and subgroups compositions that may have affected the direction of the decision-making process in order to avoid future meeting problems.

- The Keyword Extraction module is responsible for the automatic discovery of topics from submitted contributions, producing a set of keywords with their corresponding relevance score for each participant. The resulting sets represent 
the Extracted Keywords (or Topics) indicator that can be analyzed to identify subgroups compositions or isolated participants that may be the symptoms of the Feuding Factions or the Dominant Spaces syndromes that would decrease the effectiveness of the collaborative process. Furthermore the Extracted Keywords indicator reveals the level of idea generation, discussion, the level of similarity between proposed solutions or the level of convergence/divergence towards a common problem-solving model, as discussed in the previous chapters of this document. The Keyword Extraction module is coordinated by the Reasoner module that starts its execution once the coordinator selects the option to stop the fetching phase in case the meeting session has finished or having a break, since partial or none contributions would produce unreliable relevance scores. In fact, ideas can be developed during all the stages of the collaborative problem-solving process, either during the first phases or right in the middle of it. Extracting topics from single samples of the collaboration data stream would not consider concepts and notions that can be discussed in the subsequent stages of the meeting session. The natural approach is to examine the linear evolution of the collaborative process and not only the collections of fragments that represent the discrete samples generated from the Reasoner module. The implemented supervised approach used by the module takes advantage of a large training set constituted by almost 300 scientific articles from various disciplines, including Computer Science, Mechanical Engineering, and Electrical Engineering

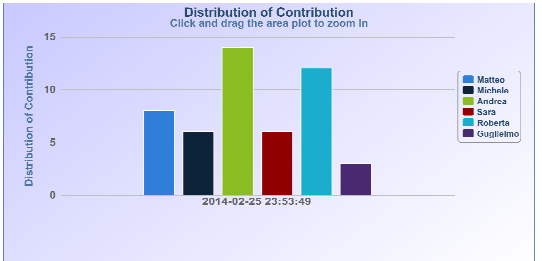

(a) distribution of contribution visualization

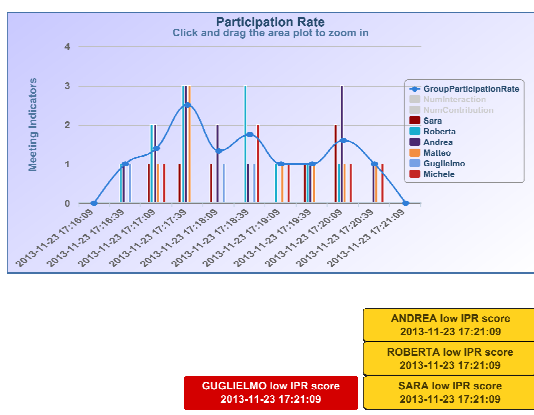

(c) Participation rate visualization

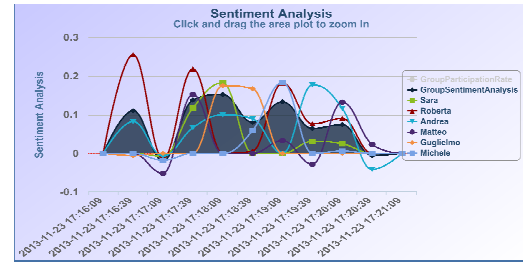

(b) Spline chart sentiment analysis visualization

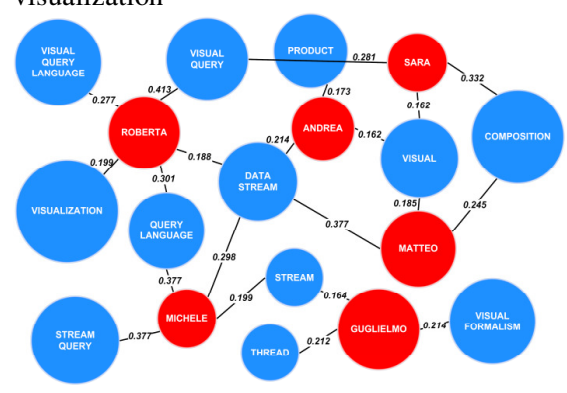

(d) Keyword extraction visualization

Fig 2. Examples of RegalMiner visualizations

- The Web Visualization module provides a computer-supported, interactive, visual representation of the data collected, managed, analyzed and processed by 
RegalMiner in order to amplify the end-user cognition of the ongoing collaborative process. All the visualization are characterized by some common features such as interactive live updates, zoomable area chart, animations, pop up tooltips for detailed information and different colors to distinguish different series.

\section{Concluding Remarks}

In this paper, we have outlined the main components of a Collaboration and Decision Making Suite specifically tailored to virtual enterprises adopting service-oriented business models and functional engineering, in which the ability to collaboratively manage huge data streams is a key factor. The platform is currently being validated in the context of an EU project by real users in the manufacturing industries, and preliminary feedbacks show the usefulness of the proposed tools, in particular the possibility to manage ad-hoc collaborations and to monitor and mine the collaborations themselves.

Acknowledgments. This work has been supported by the FP7 IP project SmartVortex Scalable Semantic Product Data Stream Management for Collaboration and Decision Making in Engineering. The authors would like to thanks Emanuela Bauleo and Serena Carnevale for the realization of the V2QT ECC.

\section{References}

1. Aggarwal, C.C., Zhai, C. (eds.): Mining Text Data. Springer (2012)

2. Bernstein, A.: How can cooperative work tools support dynamic group process? Bridging the specificity frontier. In: Proc. 2000 ACM Conference on Computer Supported Cooperative Work

3. Biehl, J.T., Czerwinski, M., Smith, G., Robertson, G.G.: Fastdash: a visual dashboard for fostering awareness in software teams. In: Proc. CHI'07

4. Dourish, P., Bellotti, V.: Awareness and coordination in shared workspaces. In: Proc. 1992 ACM Conference on Computer-supported Cooperative Work.

5. Dumas, M., van der Aalst, W., ter Hofstede, A.: Process-Aware Information Systems. Wiley Online Library (2005)

6. Ellis, C.A., Barthelmess, P., Chen, J., Wainer, J.: Person-to-person processes: Computersupported collaborative work. Process-Aware Information Systems p. 37 (2005)

7 Esuli, A., Sebastiani, F.: SentiWordNet: A Publicly Available Lexical Resource for Opinion Mining. In: Proceedings of Language Resources and Evaluation (LREC) (2006)

8 Janeiro, J., Lukosch, S., Radomski, S., Johanson, M., Mecella, M., Larsson, J.: Supporting elastic collaboration: integration of collaboration components in dynamic contexts. In: Proc. 5th ACM SIGCHI symposium on Engineering interactive computing systems (2013)

9 Malagoli, A., Leva, M., Kimani, S., Russo, A., Mecella, M., Bergamaschi, S., Catarci, T.: Visual query specification and interaction with industrial engineering data. In: Advances in Visual Computing - 9th International Symposium, ISVC 2013, Proceedings - Part II. LNCS 8034, Springer (2013)

10 Miller, G.: WordNet: An on-line lexical database. International Journal of Lexicography (Special Issue) 3, 235-312 (1990) 